(E) SOLAR SYSTEM \& PLANETARY SYSTEMS 


\title{
AND THE SOLAR ATMOSPHERE
}

\author{
A.I. POLAND \\ Laboratory for Astronomy and Solar Physics, \\ NASA Goddard Space Flight Center, Greenbelt, Maryland, USA \\ and \\ V. DOMINGO \\ Space Science Department of ESA, ESTEC, Noordwijk, The Netherlands
}

\begin{abstract}
The Solar and Heliospheric Observatory, Soho, is a joint venture of ESA and NASA. The main objectives of Soho are: a) the study and understanding of solar coronal phenomena; and b) the study of the solar structure and interior dynamics from its core to the photosphere. The primary goals of the coronal and solar wind studies are to understand the coronal heating mechanism and its expansion into the solar wind.

These goal will be achieved both by remote sensing of the solar atmosphere with high resolution spectrometers and telescopes and by "in situ" measurement of the composition and energy of the resulting solar wind and the energetic particles that propagate through it. The structure and interior dynamics will be studied by helio-seismological methods and the measurement of solar irradiance variations. The Soho spacecraft will be three-axis stabilized and located in a halo orbit around the L1 Lagrangian point (approximately $1 \%$ of the distance from the Earth to the Sun). It is currently scheduled for launch in July 1995.
\end{abstract}

\section{Introduction}

The Solar and Heliospheric Observatory, Soho, is a space mission that forms part of the Solar-Terrestrial Science Program (STSP), developed in a collaborative effort by the european Space Agency, ESA, and by the National Aeronautics and Space Administration, NASA, of the USA.

The STSP, in turn, forms part of ESA's long term science plan known as "Space Science: Horizon 2000" and of NASA's collaborative International Solar-Terrestrial Program (ISTP) with ESA and with ISAS (Institute of Space and Astronautical Science, Japan).

This paper presents an overview of the Soho mission in terms of its overall scientific objectives and its complement of instruments.

\section{Scientific Objectives}

The Sun is the only star that we can study with high spacial resolution. For example, we can observe images of the Sun and use them to study plasma processes occurring in different parts of its atmosphere. For phenomena occurring in the outer atmosphere (outside the photosphere) we are frequently able to determine many aspects of the interactions between various physical processes such as magnetic field 
changes, heating, material flow, conduction, and radiation. An important aspect of these processes is the expansion of the solar atmosphere in the form of a wind (solar wind) that blows past the Earth and most of the solar system. We can study this solar wind "in situ" by means of spacecraft that are outside of the Earth's magnetosphere.

The source region of the solar wind is the layers above the photosphere which cannot be studied from ground observatories for two main reasons. One is that they are so tenuous with respect to the photosphere that any emission that they produce at visible wavelengths cannot be seen against the photospheric background. However, some features in the corona can be observed from the Earth during total solar eclipses when the moon, for a few minutes, blocks out the solar disk. Another reason for not being able to study these regions from the ground is that they are at higher temperatures than the photosphere and therefore produce emission lines due to higher levels of ionization and excitation of the atoms. These lines are generally emitted in the ultra-violet and X-ray regions of the spectrum, to which the Earth's atmosphere is opaque. Thus, the outer layers of the solar atmosphere are not generally observable from the ground.

Previous observations of the Sun from space have been performed by NASA's Orbiting Solar Observatory (OSO) satellites during the 1960's and early 1970's, the Apollo Telescope Mount (ATM) on Skylab during 1972 and 1973, and with the Solar Maximum Mission (SMM) during the 1980's. Many of the observations made by the OSO's and ATM provided the basis for our current understanding of the outer solar atmosphere. The SMM observations further advanced our knowledge, but the experiments were tailored to study solar flares. An important conclusion based on the observations from these previous satellites is that: to understand the processes occurring in the outer solar atmosphere we need simultaneous, high spatial resolution images of the Sun at many wavelengths with a relatively rapid ( $\sim 1$ minute or less) time resolution.

Soho is a solar observatory devoted to furthering our understanding of the outer solar atmosphere and the solar wind. To accomplish this Soho will carry a set of telescopes that will study phenomena that are initiated by processes that commence below the photosphere, and propagate through the photosphere, chromosphere, and the transition region into the corona. These instruments are designed to investigate problems such as how the corona is heated and how it is transformed into the solar wind that blows past the Earth at $400 \mathrm{~km} / \mathrm{s}$. To do so it will have spectrometers that will allow the detailed study of the emission and absorption lines produced by the ions present in the different regions of the solar atmosphere. From this information it will be possible to determine densities, temperatures and velocities in the changing structures. These measurements are complemented by the "in situ" study of the composition and energies of the solar wind that results from the coronal structures that have been observed by the telescopes. This is done with the help of particle detectors carried by Soho that sample the solar wind as it passes through it. Soho will thus greatly enhance our knowledge of the solar wind and its source region.

While the solar interior is the region where the kinetic and magnetic energy that drives the outer atmosphere and solar wind is generated, almost no direct 
information can be obtained about any region below the photosphere. The neutrinos that are generated by the nuclear reactions that take place in the core are the only direct radiation that reaches us from anything that is below the photosphere. But a relatively new technique, helio-seismology, has developed in the last two decades that allows us to study the stratification and certain dynamical aspects of the solar interior. It uses the study of the acoustic and gravity waves that propagate through the interior of the Sun and can be observed as oscillatory movements of the photosphere. An analysis of these oscillations allows us to determine the characteristics of the resonant cavities in which they resonate, much in the same way as the Earth's seismic waves are used to determine the structure of the Earth's interior.

To study the solar interior Soho will carry a complement of instruments whose aim is to study the oscillations at the solar surface by measuring the velocity oscillations via the Doppler effect and by measuring the oscillating changes in intensity that the pressure and gravity waves produce. The study of such oscillations require both high resolution imaging and long uninterrupted time series of observations. In addition, because it is paramount to understand the structure of the Sun in relation to the oscillation measurements, the total solar irradiance, or solar constant, and its variations will be measured.

The Soho satellite will thus enable us to study: the structure, chemical composition, and dynamics of the solar interior, the structure (density, temperature and velocity fields) and dynamics of the outer solar atmosphere, and the solar wind and its relation to the solar atmosphere.

\section{Instrumentation}

The investigations selected for the Soho satellite are listed in Table I. They can be divided by their areas of research into three main groups: helio-seismology, solar atmospheric remote sensing, and "in situ" solar wind measurements.

The helio-seismology investigations primarily aim at the study of those parts of the solar oscillations spectrum that cannot be obtained from the ground. The required sensitivity for observing the very low modes $(l \lesssim \sim 5)$ and the very high modes $(l \gtrsim \sim 200)$ is difficult to achieve from the ground because of noise effects introduced by the Earth's diurnal rotation for the low modes, and the transparency and seeing fluctuations of the Earth's atmosphere for the high modes. GOLF and VIRGO aim primarily at the study of the very core of the Sun; for that they have to study oscillations of very low frequencies with very high sensitivity. MDI will observe the whole oscillation spectrum, and will be unique in its study of the upper degree modes that carry information about the composition and dynamics of the outer boundary layer. The three investigations will provide important information about the time varying phenomena in the interior of the Sun.

The Global Oscillations at Low Frequencies (GOLF) investigation will perform uninterrupted velocity oscillation and magnetic field measurements of the full solar disk, spatially unresolved, with extremely high sensitivity $(\$ 1 \mathrm{~mm} / \mathrm{s}$ and 1 milligauss). To determine the velocity, the Doppler effect is measured by the resonant scattering technique. 
Table I. SOHO Investigations

\begin{tabular}{|c|c|c|c|}
\hline Investigation & Principal Inv. & Measurements & Technique \\
\hline $\begin{array}{l}\text { Global } \\
\text { Oscillations at } \\
\text { Low Frequencies } \\
\text { (GOLF) }\end{array}$ & $\begin{array}{l}\text { A. Gabrie1, IAS, } \\
\text { Verrieres-le- } \\
\text { Buisson, France }\end{array}$ & $\begin{array}{l}\text { Global Sun } \\
\text { Velocity and } \\
\text { Magnetic field } \\
\text { oscillations } \\
1=0-4\end{array}$ & $\begin{array}{l}\text { Na-vapor } \\
\text { resonant cell, } \\
\text { Doppler shift } \alpha \\
\text { circular } \\
\text { polarization }\end{array}$ \\
\hline $\begin{array}{l}\text { Variability of } \\
\text { solar irradiance } \\
\text { (VIRGO) }\end{array}$ & $\begin{array}{l}\text { C. Frolich } \\
\text { PMOD/WRC, Davos, } \\
\text { Switzerland }\end{array}$ & $\begin{array}{l}\text { Low degree }(1=0 \\
7) \text { irradiance } \\
\text { oscillations and } \\
\text { solar constant }\end{array}$ & $\begin{array}{l}\text { Global Sun and } \\
\text { low resolution } \\
\text { (12 pixels) } \\
\text { imaging, active } \\
\text { cavity } \\
\text { radiometers }\end{array}$ \\
\hline $\begin{array}{l}\text { Michelson } \\
\text { Doppler Imager } \\
\text { (MDI/SOI) }\end{array}$ & $\begin{array}{l}\text { P.H. Scherrer, } \\
\text { Stanford Univ. } \\
\text { USA }\end{array}$ & $\begin{array}{l}\text { Velocity } \\
\text { oscillations, } \\
\text { high degree } \\
\text { modes (up to } \\
1=4500 \text { ) }\end{array}$ & $\begin{array}{l}\text { Doppler shift } \\
\text { with Fourier } \\
\text { tachometer, } 4 \text { \& } \\
1.5 \text { arc sec } \\
\text { resolution }\end{array}$ \\
\hline
\end{tabular}

Solar Wind "In Situ"

\begin{tabular}{|c|c|c|c|}
\hline Investigation & Principal Inv. & Measurements & Technique \\
\hline $\begin{array}{l}\text { Charge Element } \\
\text { and isotope } \\
\text { analysis } \\
\text { (CELIAS) }\end{array}$ & $\begin{array}{l}\text { D. Hovestadt } \\
\text { MPE, Garching, } \\
\text { Germany }\end{array}$ & $\begin{array}{l}\text { Energy } \\
\text { distribution } \& \\
\text { composition. } \\
0.1-1000 \mathrm{keV} / \mathrm{e}\end{array}$ & $\begin{array}{l}\text { Electrostatic } \\
\text { deflection, } \\
\text { time-of-flight } \\
\text { measurements } \& \\
\text { solid state } \\
\text { detectors }\end{array}$ \\
\hline & $\ldots . . . .$. & $\ldots$ & $\ldots-\ldots-\ldots$ \\
\hline $\begin{array}{l}\text { Suprathermal and } \\
\text { energetic } \\
\text { particle } \\
\text { analyzer } \\
\text { (CoSTEP) }\end{array}$ & $\begin{array}{l}\text { H. Kunow } \\
\text { Univ. Kiel } \\
\text { Germany }\end{array}$ & $\begin{array}{l}\text { Energy } \\
\text { distribution \& } \\
\text { composition, } \\
\text { ions } 1.2-330 \\
\mathrm{MeV} / \mathrm{n} \text { electrons }\end{array}$ & $\begin{array}{l}\text { Solid state, a } \\
\text { plastic and } \\
\text { crystal } \\
\text { scintillator } \\
\text { detector }\end{array}$ \\
\hline $\begin{array}{l}\text { Energetic } \\
\text { particle analy. } \\
\text { (ERNE) }\end{array}$ & $\begin{array}{l}\text { J. Torsti } \\
\text { Univ. Turku, } \\
\text { Finland }\end{array}$ & $0.06-25 \mathrm{MeV}$ & telescopes. \\
\hline
\end{tabular}

Solar Ultraviolet emitted radiation (SUMER)
K. Wilhelm MPAE, Lindau Germany
Plasma flow characteristics (T, $\rho$, velocity) chromosphere corona
Normal incidence spectrometer, 50-160 nm, spectr. res. 2 40,000 ang. res. 1. $2-1.5^{\prime \prime}$
Coronal

Diagnostics spectrometer (CDS)
B.E. Patchett

RAL Chilton

England
Temperature and $\rho$ : transition region \& corona
Grazing incidence spectrometer 17 $80 \mathrm{~nm}$ spectr. res. 5000. ang res. 2" 
Table I: continued

Solar Atmosphere Remote Sensing

\begin{tabular}{|c|c|c|c|}
\hline Investigation & Principal Inv & Measurements & Technique \\
\hline $\begin{array}{l}\text { Extreme-ultra- } \\
\text { violet imaging } \\
\text { telescope } \\
\text { (EIT) }\end{array}$ & $\begin{array}{l}\text { J.P. Delaboudin- } \\
\text { iere IAS, } \\
\text { Verrieres-le- } \\
\text { Buisson, France }\end{array}$ & $\begin{array}{l}\text { Evolution of } \\
\text { chromospheric \& } \\
\text { coronal } \\
\text { structures }\end{array}$ & $\begin{array}{l}\text { Images in HeII, } \\
\text { FeIX, FeXII, \& } \\
\text { FeXV }\end{array}$ \\
\hline $\begin{array}{l}\text { U1tra-violet } \\
\text { coronagraph } \\
\text { spectrometer } \\
\text { (UVCS) }\end{array}$ & $\begin{array}{l}\text { J.L. Kohl, SAO } \\
\text { Cambridge, Mass. } \\
\text { USA }\end{array}$ & $\begin{array}{l}\text { Electron \& ion } \\
T, \rho \text {, and vel. } \\
\text { in corona ( } 1.3- \\
\text { lORo) }\end{array}$ & $\begin{array}{l}\text { Profiles and/or } \\
\text { intensity of } \\
\text { several spectral } \\
\text { EUV lines }\end{array}$ \\
\hline $\begin{array}{l}\text { White Light \& } \\
\text { spectrometric } \\
\text { coronagraph } \\
\text { (LASCO) }\end{array}$ & $\begin{array}{l}\text { G. Brueckner NRL } \\
\text { Washington } \\
\text { USA }\end{array}$ & $\begin{array}{l}\text { Evolution, mass, } \\
\text { momentum, and } \\
\text { energy transport } \\
\text { in the corona } \\
\text { (1.1-30Ro) }\end{array}$ & $\begin{array}{l}1 \text { internal and } \\
\text { externally } \\
\text { occulted } \\
\text { coronagraphs. } \\
\text { Spectrometer. }\end{array}$ \\
\hline $\begin{array}{l}\text { Solar Wind } \\
\text { Anisotropies } \\
\text { (SWAN) }\end{array}$ & $\begin{array}{l}\text { J.L. Bertaux, SA } \\
\text { Verriers-le- } \\
\text { Buisson France }\end{array}$ & $\begin{array}{l}\text { Solar wind mass } \\
\text { flux } \\
\text { anisotropies }\end{array}$ & $\begin{array}{l}\text { Scanning } \\
\text { telescopes with } \\
\text { hydrogen abs. } \\
\text { cell for Ly } \alpha\end{array}$ \\
\hline
\end{tabular}

A resonant cell filled with sodium vapor in a strong magnetic field enables, by the Zeeman effect, the selective absorption of light in the two wings of the solar D lines. A comparison of the intensity on both sides of the line leads to an extremely sensitive determination of the velocity.

The Variability of Irradiance and Gravity Oscillations (VIRGO) Investigation is an experiment to study the solar irradiance variability and oscillations. The instrumentation of VIRGO comprises two active cavity radiometers observing the total irradiance, the sun-photometers measuring the spectral irradiance at three wave-lengths in the near ultraviolet, the visible and near infrared $(335,550$ and $865 \mathrm{~nm}$ ). In addition, narrow band radiance measurements (at $500 \mathrm{~nm}$ ) are carried out with 12 resolution elements on the solar disc by a high-precision luminosity oscillation imager.

The Michelson Doppler Imager (MDI) will measure velocity oscillations on the surface of the Sun with high angular resolution (4 and $1.5 \mathrm{arcsec}$ ). It uses two solid Michelson interferometers as the final elements of a interferometers as the final elements of a tunable narrow-bandpass filter. Prefiltering is accomplished by a blocking filter and a Lyot filter. The Michelson interferometers are tuned by halfwaveplates. Doppler shifts are determined by measuring intensities at four points along the line profile. The technique yields line-shift estimates with a linear response 
over a range of circa $4000 \mathrm{~m} / \mathrm{s}$.

The solar atmosphere remote sensing investigations are carried out with a set of telescopes and spectrometers that will produce the data necessary to study the dynamic phenomena above the chromosphere. The plasma will be studied by spectroscopic measurements and high resolution images at different levels of the solar atmosphere. Plasma diagnostics obtained with these instruments will provide temperature, density, and velocity measurements of the material in the outer solar atmosphere.

The SUMER, CDS, and EIT experiments are highly complementary in terms of the scientific objectives they can achieve. CDS obtains data on hotter lines, while SUMER obtains somewhat cooler lines, with an overlap between 50 and 80 $\mathrm{nm}$. They both obtain spectra along a spatial line on the Sun, building up a two dimensional image by moving the Sun's image across a slit in a very short time (on the order of seconds). CDS has a broader spectral range at any one time, but cannot obtain line profiles. SUMER, on the other hand, has a high spectral resolution but a limited simultaneous range. Thus, CDS is better suited for density and temperature diagnostics, while SUMER is better suited for velocity measurements. Finally, EIT provides high resolution images of the whole Sun at several temperatures (HeII $30.4 \mathrm{~nm} \sim 60,000 \mathrm{~K}$, FeIX $17.1 \mathrm{~nm} \sim 3 \times 10^{6} \mathrm{~K}$, FeXII $19.5 \mathrm{~nm}, \sim 1.6 \times 10^{6} \mathrm{~K}$, and FeXV $28.4 \mathrm{~nm} \sim 3 \times 10^{6} \mathrm{~K}$ ), thus providing the morphological context of the spectral observations.

While the CDS, SUMER and EIT primarily observe on the solar disk, the UVCS, LASCO, and SWAN experiments make observations in the solar corona. UVCS observes line profiles in the ultra-violet using Doppler dimming and broadening to determine material velocity and temperature. LASCO uses white light measurements to obtain electron density in the corona and spectral observations of emission lines and Fraunhofer lines to determine the hot coronal temperatures and electron temperature respectively.

The SWAN experiment monitors the large scale properties of the solar wind expansion, and in particular the latitude distribution of the solar wind mass flux from equator to pole, and the time variations of this distribution. It does so with Lyman alpha sky maps of interplanetary emission, obtained with an Hydrogen cell and an appropriate optical scanning system.

The instruments to measure "in situ" the composition will determine the elemental and isotopic abundances, the ionic charge states and velocity distributions of ions originating in the solar atmosphere. The energy ranges covered will allow the study of the processes of ion acceleration and fractionation under the various conditions that cause their acceleration from the "slow" solar wind through solar flares. The CELIAS instruments concentrate on "lower" energy process while ERNE and COSTEP put their main emphasis on measurements into the suprathermal energy range.

The CELIAS instrument consists of three sensors, all making use of the timeof-flight technique (TOF). The CTOF determines the elemental composition, the charge state distribution, the temperatures and the velocities of the more abundant solar wind ions from helium to iron. The MTOF determines the elemental and isotopic composition of solar wind ions with a mass resolution $(\mathrm{M} / \Delta m)$ better than 
100. Finally, the STOF determines mass, charge state and energy of suprathermal and low energy solar energetic particles from hydrogen to the iron group.

The COSTEP and ERNE investigation teams have formed a consortium in which they have defined five sensors that are best suited within the available resources to analyze the composition of the solar suprathermal and energetic particles above the range covered by CELIAS. All the sensors are basically composed of solid state particle detector telescopes. All together they measure, the electron energy spectrum between $60 \mathrm{keV}$ and $5 \mathrm{MeV}$, the proton flux between $60 \mathrm{keV}$ and $53 \mathrm{MeV}$, the energy variation of the isotopic composition of ions with energy between 1.4 $\mathrm{MeV} / \mathrm{n}$ and $53 \mathrm{MeV} / \mathrm{n}$ for NI. The charge composition determination extends ups to $540 \mathrm{MeV} / \mathrm{n}$ for elements in the $\mathrm{Ni}$ region.

In summary, the coronal remote sensing and the "in situ" experiments on Soho will provide a comprehensive data set to study the solar wind heliosphere. The solar imagers and spectrographs will allow the study of the morphology, magnetic structure, and heating and particle acceleration processes occurring at the Sun. At the same time it will be possible to make direct measurements of the particle composition and energy spectrum in the solar wind with the particle experiments.

\section{Spacecraft and Orbit}

The SOHO spacecraft will be three-axis stabilized and pointing to the sun within an accuracy of 10 aresec and a pointing stability of 1 arcsec per 15 minutes. The Total mass will be about $1350 \mathrm{~kg}$ and $750 \mathrm{~W}$ power will be provided by the solar panels. The payload will weigh about $650 \mathrm{~kg}$ and consume $350 \mathrm{~W}$ in orbit.

Soho is planned to be launched in March 1995 and will be injected in a halo orbit around the L1 Sun-Earth Lagrangian point, about $1.5 \times 10^{6} \mathrm{~km}$ sunward from the Earth. The halo orbit will have a period of 180 days and has been chosen because, 1) it provides a smooth Sun-spacecraft velocity change throughout the orbit, appropriate for helio-seismology, 2) is permanently outside of the magnetosphere, appropriate for the "in situ" sampling of the solar wind and particles, and 3) allows permanent observation of the Sun, appropriate for all the investigations.

\section{Operation, Data and Ground Systems}

The Soho telemetry will be received by ground stations of NASA's Deep-Space Network (DSN) during three short $(1.3 \mathrm{hr})$ and one long $(8 \mathrm{hr})$ periods per day. Scientific data acquired outside these periods will be stored on magnetic tape onboard the spacecraft and transmitted to ground during the short ground contact periods. The Soho payload will produce a continuous stream of 40 kilobits/s, however the bitrate will be increased by $160 \mathrm{kilobits} / \mathrm{s}$ whenever the solar oscillations imaging instrument is operated in its high bit-rate mode. This will happen either daily during the 8-hr periods or during dedicated campaigns when the satellite will have 24 hour DSN coverage. The campaigns will be organized to provide approximately 2 -months un-interrupted observations by the solar oscillation imaging instrument.

An Experiment Operations Facility (EOF), located at NASA's Goddard Space 
Flight Center, will be used to coordinate and plan the scientific operations of the payload. Its main task will be to organize in particular the real time operation of the payload and control of the solar remote sensing imaging and spectrometric instruments during the daily 8 -hr ground contact interval. ESA also intends to issue an Announcement of Opportunity to invite proposals for a second science operations center in Europe. Moreover, the large amount of solar oscillations imaging data produced by MDI will be the object of a specialized data facility.

\section{Coordinated Research}

The Soho payload has been conceived as an integrated package that requires coordinated operation and data analysis between the investigations aboard to achieve its scientific aims. The experiment Operation Facility will be the focal point for these coordinated activities. The EOF will also provide a focus for the cooperation with ground observatories. Soho will also collaborate with other space missions. For instance WIND and Cluster, when outside the magnetosphere, will provide solar plasma parameters what will complement the coronal and solar wind measurements of Soho. When Cluster is inside the magnetosphere Soho will be a useful monitor for the conditions of the environment external to the magnetosphere.

In the case of Cluster there is another aspect of cooperation that will occur. Considerable mutual insights and cross-fertilization in the area of plasma physical processes is expected from a coordinated study of the problems investigated individually by each of the two missions. As part of an ESA sponsored workshop, several working groups formulated common scientific objectives and cross-fertilization aspects between Cluster and Soho ESA, 1985. Their recommendations point out that most of the processes studied by the one of the STSP components - Soho or Cluster - have counterparts, - or at least analogues, to be studied by the other component. Joint studies by Cluster and Soho will illustrate the roles of the different parameter regimes and the limits of analogy. This process is particularly important to our attempts to extrapolate and apply knowledge gained in solar-system studies to. remote astrophysical objects.

\section{References}

Jointly released by ESA (SCI87/1) and NASA (AO-OSSA-1-87): 1987, Cluster and SOHO, announcement of Opportunity

ESA SP-235: 1985, Proceedings of an ESA sponsored workshop on "Future Missions in Solar, Heliospheric and Space Plasma Physics", Garmisch-Partenkirchen 